\title{
Electron Spin Resonance on Carbon Nanotubes-Polymer Composites
}

\author{
MIRCEA CHIPARA, ${ }^{1}$ JEFFREY M. ZALESKI, ${ }^{1}$ DAVID HUI, ${ }^{2}$ CHUNSHENG DU, ${ }^{3}$ NING PAN ${ }^{3}$ \\ ${ }^{1}$ Indiana University, Department of Chemistry, Bloomington, Indiana \\ ${ }^{2}$ Department of Mechanical Engineering, University of New Orleans, New Orleans, Louisiana \\ ${ }^{3}$ Nanomaterials in the Environment, Agriculture and Technology (NEAT), University of California, \\ Davis, California
}

Received 1 May 2005; revised 12 July 2005; accepted 18 August 2005

DOI: 10.1002 / polb.20647

Published online in Wiley InterScience (www.interscience.wiley.com).

\begin{abstract}
Electron spin resonance investigations on styrene-polyisoprene-polystyrene block copolymer loaded with various amounts of multiwalled carbon nanotubes are reported. The temperature dependence of resonance line parameters in the range 290-425 K was analyzed. It was proved that the main resonance line represents a bottleneck of localized and delocalized electrons residing on carbon nanotubes. The temperature dependence of the $g$-factor and double integral of resonance spectra confirmed this interpretation. The temperature dependence of the resonance linewidth of composites containing various concentrations of nanotubes was explained by the thermally activated narrowing of the resonance spectra. (02005 Wiley Periodicals, Inc. J Polym Sci Part B: Polym Phys 43: 3406-3412, 2005
\end{abstract}

Keywords: block copolymers; carbon nanotube; composites; electron spin resonance (ESR); nanocomposites; polymer; styrene-isoprene-styrene

\section{INTRODUCTION}

Electron spin resonance (ESR) spectroscopy has been used to assess the quality of carbon nanotube, CNT. ${ }^{1}$ Not-annealed CNT exhibits three ESR lines ${ }^{1-18}$ a wide line located at $g$ values $>2.0023$ assigned to catalyst residues and two lines located close to $g=2.0023$ assigned to paramagnetic impurities and electrons delocalized over the conducting domains of CNTs. ${ }^{2-18}$ The annealing process reduces markedly the intensity of the wide line $^{7}$ (due to catalyst residues). However, few ESR studies ${ }^{2}$ were done on CNTs dispersed in polymeric matrixes.

$$
\text { edu) }
$$

Correspondence to: M. Chipara (E-mail: mchipara@indiana.

Journal of Polymer Science: Part B: Polymer Physics, Vol. 43, 3406-3412 (2005) @ 2005 Wiley Periodicals, Inc.
The outstanding properties of CNTs ${ }^{19-21}$ such as huge mechanical strength, high electric conductivity, excellent thermal conductivity, and huge aspect ratio fuelled much research on composite materials obtained by dispersing nanotubes into different polymeric matrixes. Light materials with tailored electrical and thermal conductivity, enhanced mechanical properties, and improved thermal stability have been reported. ${ }^{21} \mathrm{~A}$ better knowledge of the interaction between CNTs and the polymeric matrix and in particular, an accurate understanding of the role and properties of the interface between CNT and macromolecular chain is mandatory to further improve the physical properties of these composites. This paper concentrates on the ESR spectra of CNTs dispersed within a polymeric matrix, aiming for a better understanding of the interactions between nanotubes and polymeric chains. 


\section{EXPERIMENTAL}

Multiwalled carbon nanotubes (CNTs) were obtained by chemical vapor deposition, at $950{ }^{\circ} \mathrm{C}$ in a tube furnace, using $\mathrm{CH}_{4}$ as carbon source, hydrogen as gas carrier, and $\mathrm{Fe}$ deposited on $\mathrm{MgO}$ as catalyst. The as-grown nanotubes were purified by acidic $(\mathrm{HCl})$ treatment. Nevertheless, this process did not completely remove the iron catalyst (located either in the tips of nanotubes or coated by amorphous carbon). About $85 \%$ of nanotubes had a diameter of $3 \mathrm{~mm}$ and a length of $8 \mu \mathrm{m}$. Composite materials were obtained by dispersing various amounts of multiwalled carbon nanotubes within a styrene-polyisoprene-polystyrene block copolymer (SIS, from Aldrich). The block copolymer was dissolved in toluene. The CNT was introduced in the dilute solution of SIS in toluene (about $2 \%$ wt.) and the as-obtained mixture was sonicated for few days at room temperature and low sonication power density (maximum power $0.1 \mathrm{Watts} / \mathrm{m}^{3}$ ) was used to prevent polymer degradation. After homogenization, the solution was poured onto microscope slides and the solvent was evaporated at about $80{ }^{\circ} \mathrm{C}$ for $24 \mathrm{~h}$. Composites containing $0.25 \%$, $1.0 \%, 2.5 \%, 5.0 \%$, and $7.0 \%$ CNT in SIS (SIS-CNT) were obtained and investigated by ESR spectroscopy.

The ESR spectra were recorded by a Bruker spectrometer $($ EMX) operating in $\mathrm{X}$-band $(\approx 9 \mathrm{GHz})$ and equipped with a temperature accessory. The dependence of resonance line parameters on the concentration of CNT dispersed in the polymeric matrix and the temperature dependence of resonance line parameters in the temperature 300 $425 \mathrm{~K}$ are reported below.

\section{RESULTS AND DISCUSSIONS}

The ESR spectra of SIS-CNT composites are reminiscent to the ESR spectra of CNTs. ${ }^{2-18}$ The resonance spectrum of SIS-CNT composites consists of three lines. The first line is located at low magnetic field (corresponding to a $g$-factor of about 4). This weak line, which originates from catalyst residues (actually isolated high spin $\mathrm{Fe}^{3+}$ ions) is not shown as it is not related to the goals of this study. For all range of CNT concentrations and temperatures reported in this article, the resonance spectrum of SIS-CNTs is dominated by a strong and symmetric line located (see Fig. 1) at about $g=2.06$ and characterized by a resonance linewidth of the order of
$10^{2} \mathrm{G}\left(1 \mathrm{G}=10^{-4} \mathrm{~T}\right)$. Such parameters are typical for the ESR spectrum of strongly interacting localized and itinerant electrons, in the bottleneck regime. The itinerant electrons are delocalized over the conducting domains of annealed CNTs. $^{2,7}$ The symmetry of the resonance line reflects a good dispersion of nanotubes within composites and indicates that SIS-CNT composites are characterized by a low electrical conductivity. Conducting nanoparticles are responsible for an asymmetric resonance line shape (Dysonian $^{2,11,17}$ ) because of the damping of the microwave field inside the particle. A faint narrow line (shown in the inset of Fig. 1) is superimposed on the intense line. Hence, its parameters (line position, width, and amplitude) cannot be accurately estimated. The weak line was tentatively assigned to paramagnetic impurities (such as graphite).

In Figure 2 it is shown the temperature dependence of the $g$-factor for SIS-CNT composites. For an uncoupled electronic $\operatorname{spin}(s=1 / 2)$ the $g$-factor is

$$
h v=g \beta H_{\mathrm{RES}}
$$

where $h$ is the Planck's constant, $\beta$ is the Bohr magneton of the electron, $v$ is the frequency of the microwave field (kept constant during the recording of the spectrum), and $H_{\mathrm{RES}}$ is the value of the external magnetic field at which the resonance is observed (resonance field). The theoretical value for the $g$-factor of the free electron is $g_{\text {th }}$ $=2.0023 .^{2,11,17}$ The observed shift of the $g$-factor comes from several contributions. The first one represents the spin-orbit coupling. ${ }^{2,11-17}$ The second one reflects the effect of the crystalline field on the resonance line position. ${ }^{22}$ The resonance spectra of SIS-CNT composites are a convolution over all resonances corresponding to different orientations of CNTs relative to the external magnetic field. However, the observed shift of the $g$ factor from $g_{\text {th }}$ is larger than the g-factor shift predicted for the spin-orbit coupling of carbon atoms. ${ }^{22}$ The third contribution expresses the fact that the resonance spectrum is due to both localized and delocalized electrons. These electrons are strongly interacting and the system is in the so-called bottleneck state, when one type of electrons governs the relaxation mechanism. ${ }^{18}$

As it is observed from Figure 2, the g-factor increases as the temperature is increased. Such dependence suggests that the intense resonance line showed in Figure 1 is due to the competition between two interacting electron species. One 


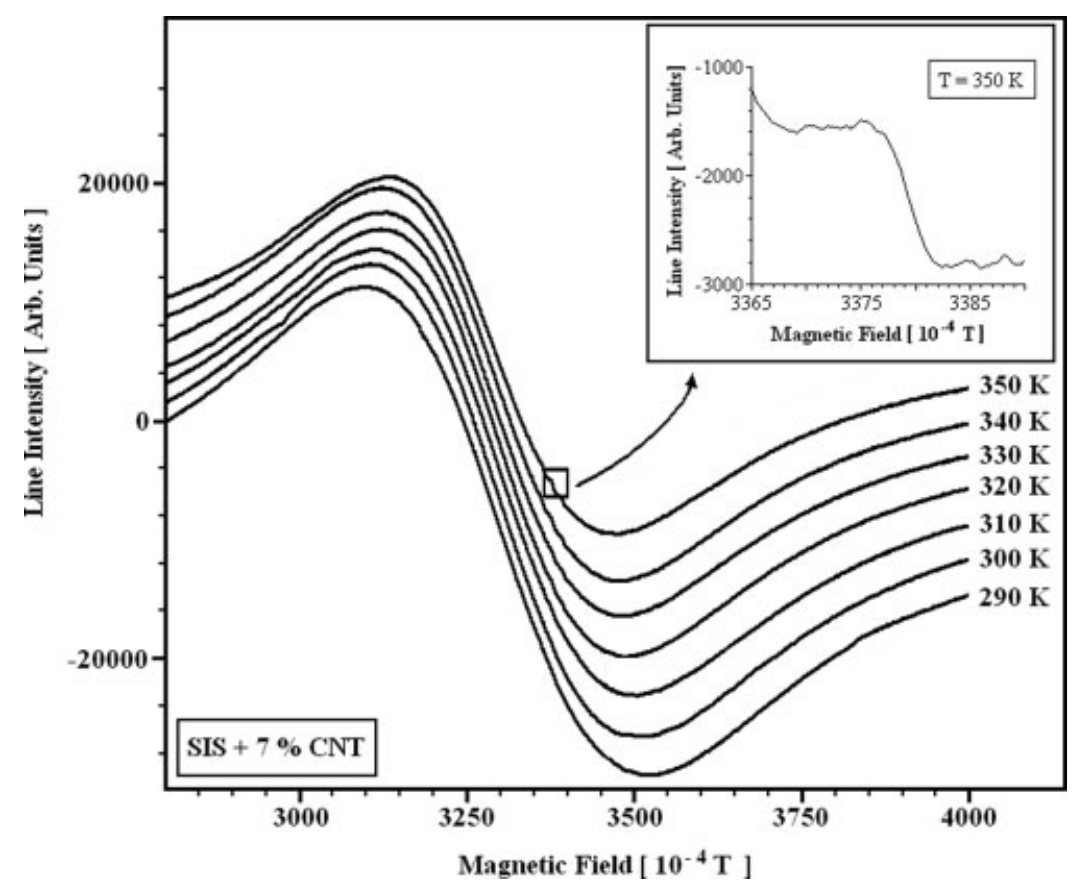

Figure 1. ESR spectra of CNT-SIS composites (containing 7 wt \% CNT), at various temperatures. The inset shows a magnified region of the spectrum that contains the faint line, as recorded at $350 \mathrm{~K}$. The amplitude of this line increases slowly as the temperature is increased.

species is located within the conducting domains of nanotubes, characterized by $g_{1}$ and a temperature-independent susceptibility. The second is a localized electron (paramagnetic) characterized by $g_{2}$. A simple Curie-like dependence describes accurately the temperature dependence of the susceptibility $\left(\chi_{2}\right)$ of localized electrons. In the bottleneck regime, the $g$ factor of the observed resonance line is ${ }^{18}$

$$
g=\frac{g_{1} \chi_{1}+g_{2} \chi_{2}}{\chi_{1}+\chi_{2}}=\frac{g_{1} \chi_{1}+g_{2}\left(\frac{K_{2}}{T}\right)}{\chi_{1}+\frac{K_{2}}{T}}
$$

where $K_{2}$ is the Curie constant $\left(\chi_{2}=K_{2} / \mathrm{T}\right)$. The increase of the $g$-factor as the temperature was increased from $100 \mathrm{~K}$ to $300 \mathrm{~K}$ has been reported in electron-beam irradiated carbon nanotubes and assigned to the exchange interaction between localized and delocalized electrons. ${ }^{18}$ The line shown in Figure 2 represents the best fit obtained by using the expression (2) for SIS loaded with $5 \%$ CNTs. For this fit the $g$-factor of the CNTs was supposed to be equal to $g_{1}=2.012$, according to literature data. ${ }^{22}$ The best fit of the temperature dependence of the $g$-factor obtained by using the expression (2) was consistent with $g_{2}=2.14$ \pm 0.02 . This value corresponds to the $g$-factor of isolated $\mathrm{Fe}^{3+}$ ions ${ }^{27}$ and reveals the effect of catalyst residues on the electronic properties of nanotubes. The Pauli susceptibility has been estimated at about $5 \times 10^{-8} \mathrm{emu} / \mathrm{g}$, in agreement with pre-

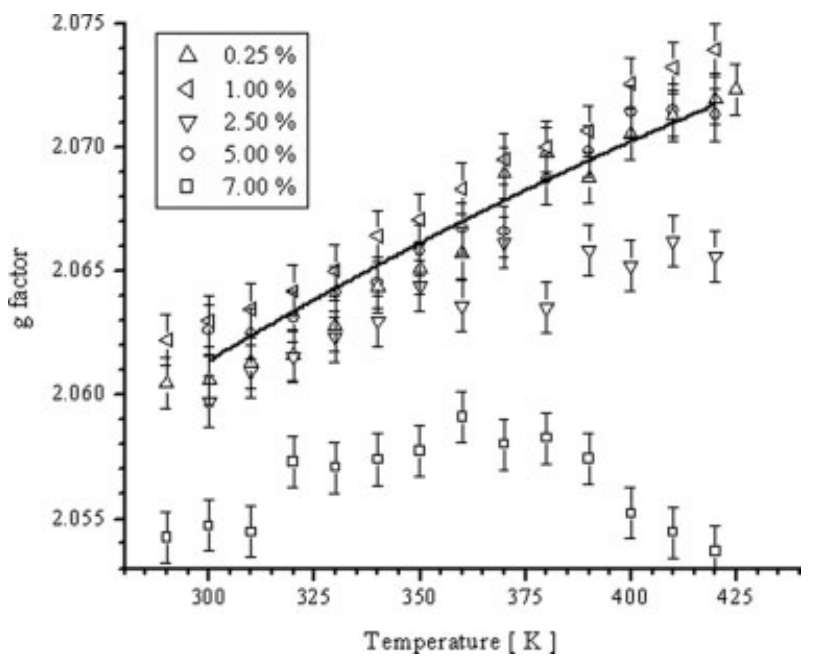

Figure 2. The temperature dependence of the $g$ factor SIS-CNT composites for different loadings with CNT. The line represents the best fit of the temperature dependence of the $g$-factor for the composite containing 5\% CNT. 


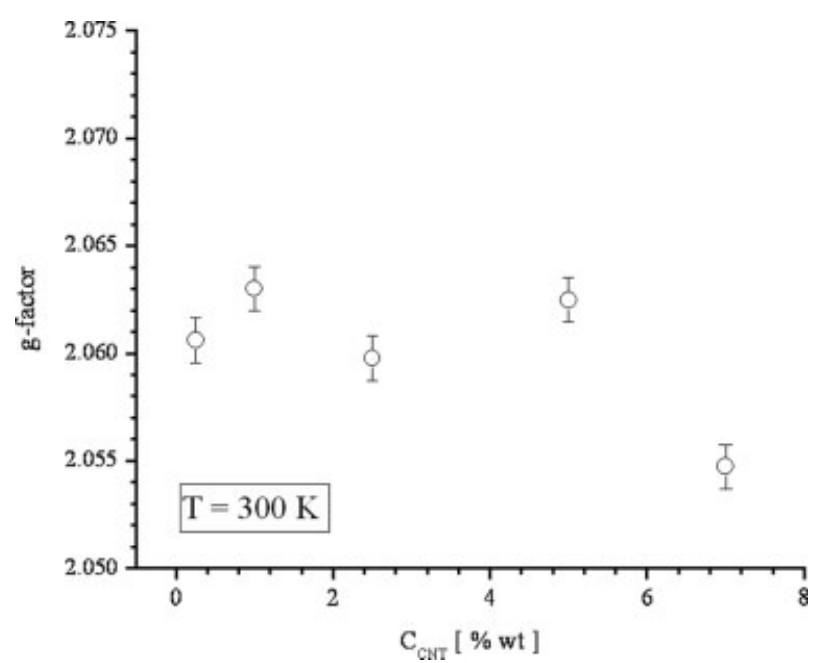

Figure 3. The dependence of the $g$ factor of SISCNT composites on the amount of CNT dispersed within the polymeric matrix, at $300 \mathrm{~K}$.

vious ESR data on CNTs. ${ }^{22}$ We suppose that in the investigated system there is a complex exchange interaction between the itinerant electrons localized on CNTs and the localized electrons located on both catalyst residues (isolated Fe ions) and other paramagnetic centers (actually radicals and/or graphite). The modification of the weight of the contribution of paramagnetic defects to the temperature dependence of the $g$-factor of nanotubes as their concentration is increased reflects the effect of the interaction between nanotubes and macromolecular chains. Three possible processes may be invoked; the first is represented by a very weak mechanical fracture of nanotubes in polymeric matrixes. The second one reflects the production of non-paramagnetic defects due to the stress felt by nanotube from the polymeric matrix. ${ }^{18}$ Such non-paramagnetic defects may modify the density of states and the Fermi level. ${ }^{18}$ Thermally activated collisions between CNTs may also explain the increase of the $g$-factor as the sample temperature is increased. CNTs collisions may either distort the structure of CNT affecting the energy levels and the electronic populations or even produce paramagnetic molecules if the CNTs are fractured. Mechanical generation of free radicals in CNTs has been reported. ${ }^{23}$ The sample with the highest amount of nanotubes shows a different behavior resulting from a more complex competition between localized and delocalized electrons. The gradual increase of the DC conductivity as the concentration of CNT dispersed in SIS is increased may also affect the dependence of the $g$-factor on temperature for the highest CNT concentration (through the Elliot mechanism ${ }^{11}$ ). The relatively scattered dependence of the $g$-factor on temperature may result from the convolution of the previously mentioned processes. The particular shape of the $g$-factor dependence on the temperature for the sample containing 7\% CNT indicates that the interactions between localized defects are greatly enhanced; accordingly the temperature dependence of localized spins is changed from a simple Curie dependence into a CurieWeiss-like dependence.

In Figure 3 it is depicted the dependence of the $g$-factor on the concentration of nanotubes. The experimental data are rather scattered. A weak tendency of the g-factor to decrease as the concentration of nanotubes is increased may be observed. This may result either from stronger exchange interactions between catalyst residues or from the increase of the DC conductivity of the composite, through Elliott mechanism. ${ }^{11}$

The temperature dependence of the resonance linewidth, $H_{\mathrm{PP}}$, is shown in Figure 4 . The resonance linewidth is governed by the relaxation of electronic spins. Two main types of processes govern the resonance linewidth, namely the spinspin interaction and the spin-lattice interactions. The intensification of exchange interactions and of the electronic jump frequencies averages the inhomogeneities of the local magnetic fields, narrowing the resonance line. In metallic systems, the Elliott mechanism ${ }^{11}$ provides an intimate relationship between the resonance linewidth,

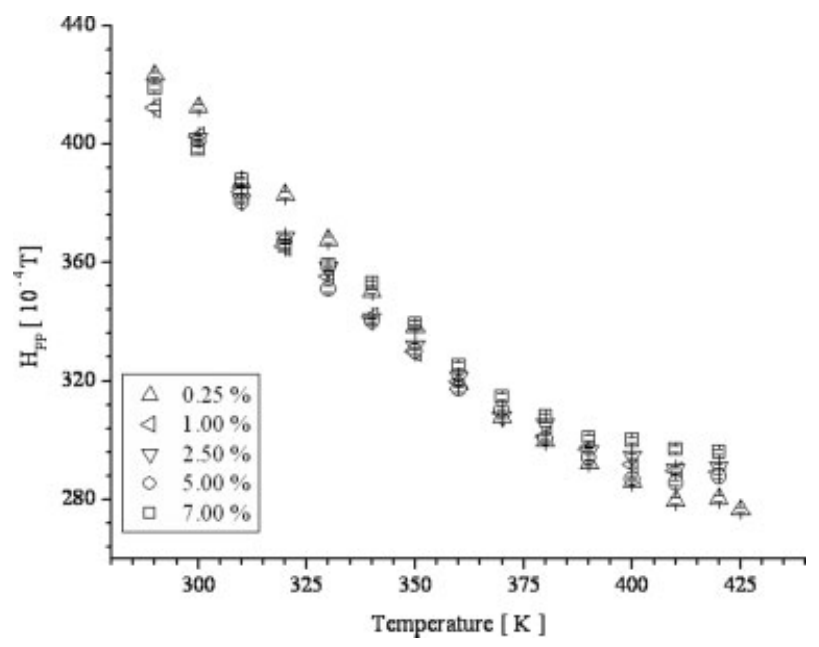

Figure 4. The temperature dependence of the resonance linewidth of CNT-SIS composites for different loadings with CNT. 


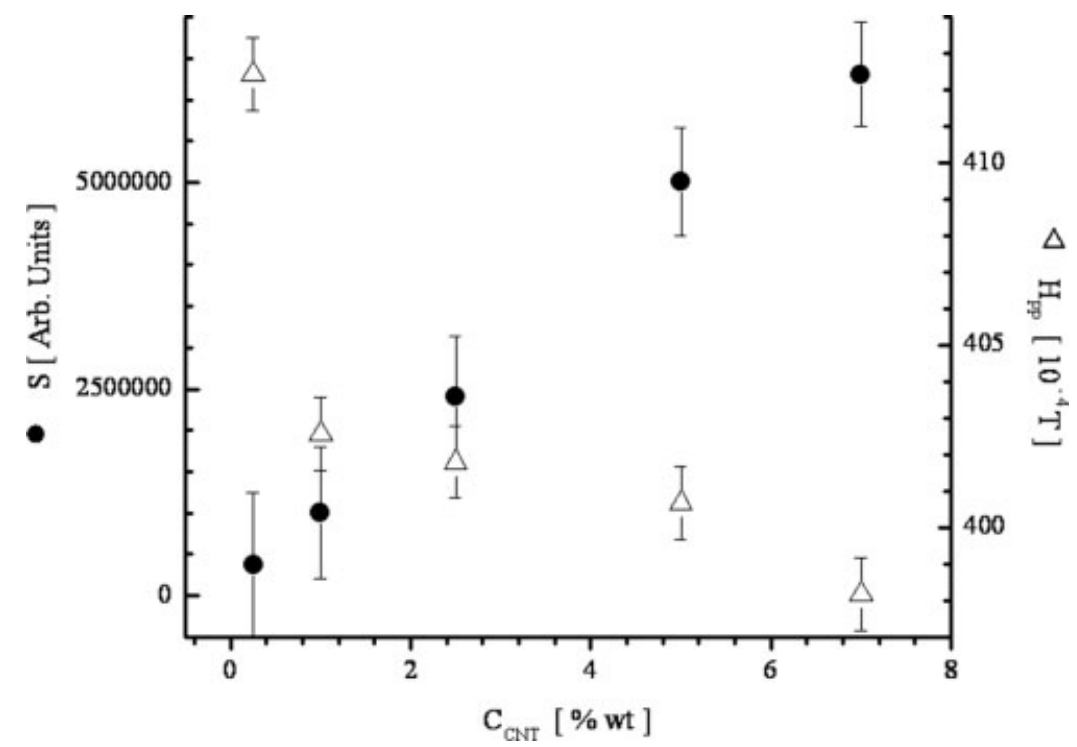

Figure 5. The dependence of the resonance linewidth (right axis, triangles) and of the double integral of the resonance spectrum (left axis, filled circles) of SIS-CNT on the amount of CNT dispersed within the polymeric matrix at $300 \mathrm{~K}$.

the $g$-factor, and the electronic relaxation processes (connected to the electrical conductivity). ${ }^{29}$ As it is observed in Figure 4, the resonance linewidth decreases as the temperature is increased, due to the enhancement of the segmental jump frequency. This amplifies the collisions between nanotubes, leads to a better averaging of the resonance line, and finally results in a narrowed resonance line. ${ }^{24}$ The temperature dependence of the resonance linewidth (in the range 290-370 K) is accurately described by an Arrhenius-like behavior

$$
H_{\mathrm{PP}}=H_{\mathrm{PP}}^{(0)} \exp -\frac{E_{\mathrm{A}}}{K_{\mathrm{B}} T}
$$

where $H_{\mathrm{PP}}^{0}$ is a constant (fitting parameter), $E_{\mathrm{A}}$ is the activation energy, and $K_{\mathrm{B}}$ the Boltzmann constant. The best fits were characterized by high correlation coefficients (over 0.98) and activation energies of the order of $3.5 \pm 0.1 \mathrm{KJ} / \mathrm{mol}$. The activation energy showed no dependence on CNTs concentration. The deviations noticed above $370 \mathrm{~K}$ are triggered by the glass transition of the polystyrene phase (which is about 370 $\mathrm{K}^{26}$ ), which is responsible for a rapid increase of styrene segmental motions. ${ }^{25,26}$ The dependence of the resonance linewidth on the CNT loading at $300 \mathrm{~K}$ is shown in Figure 5 (right axis). It is observed that the resonance linewidth decreases as the CNTs concentration is increased. This confirms the proposed mechanism for resonance linewidth based on thermally activated collisions. By increasing the nanotube concentration the collision frequency is increased and hence the resonance linewidth is narrowed.

The double integral of the resonance line, S, has been calculated according to ${ }^{24}$

$$
S=\frac{k I H_{\mathrm{PP}}^{2}}{m A}
$$

where $k$ is the resonance line shape parameter, $I$ is the amplitude of the resonance spectrum, $H_{\mathrm{PP}}$ is the peak-to-peak linewidth, $m$ is the mass of the sample (composite), and $A$ is the spectrometer's gain. Taking into account that $S$ is proportional to the susceptibility, the temperature independence of $S$ confirms that the intense resonance line originate from the severe interaction of the electrons delocalized over the conducting domains of CNTs with localized electrons (most of them belonging to isolated $\mathrm{Fe}^{3+}$ ions). The Pauli metallic susceptibility of delocalized electrons is almost temperature-independent, ${ }^{2,13,17,24}$ while the susceptibility of localized electrons obeys a Curie-like dependence. As it is observed from Figure 6, a weak temperature dependence of the spin susceptibility was observed for the samples with a low content of CNT. Figure 6 revealed that as the concentration of CNT is increased the weight of the paramagnetic contribution is increased and the temperature dependence of $S$ is enhanced. This result is consistent 


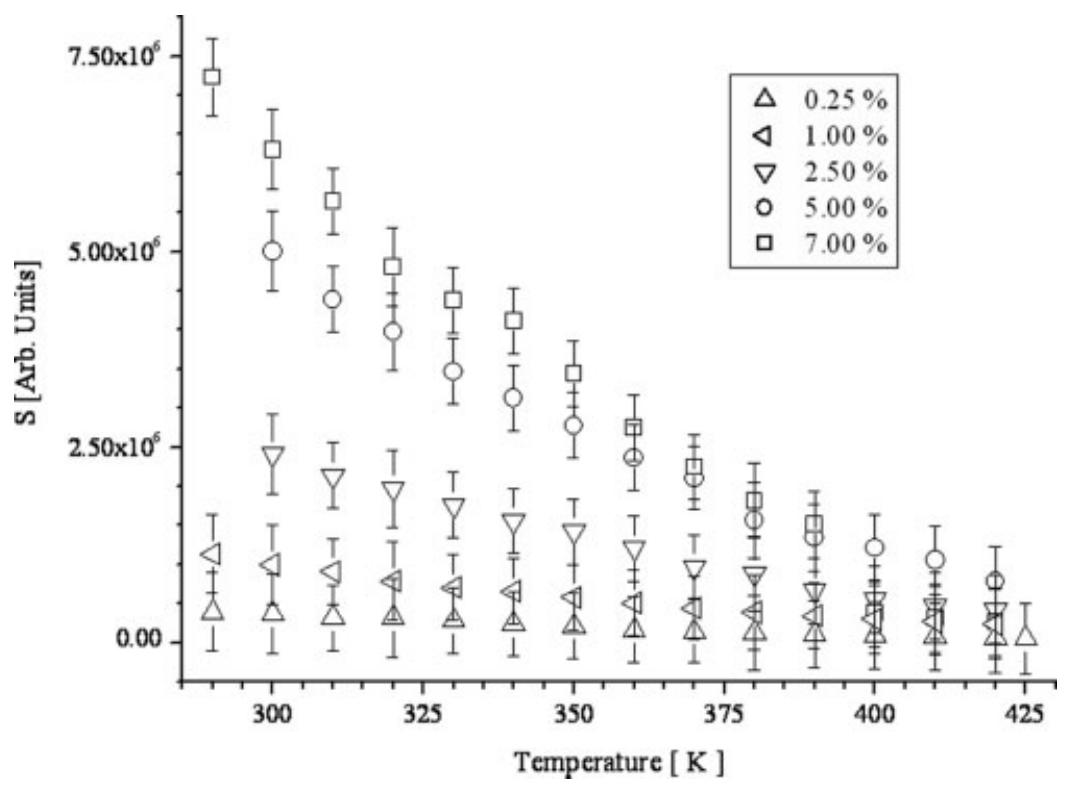

Figure 6. The temperature dependence of the double integral of the resonance line of SIS-CNT composites for different loadings with CNT.

with the spin bottleneck process, used to explain the $g$-factor dependence on temperature. Some paramagnetic defects (such as $\mathrm{Fe}^{3+}$ ions and graphite) are introduced within the composite by CNTs. However, the dispersion of nanotubes into polymeric matrixes may also generate localized electrons (paramagnetic defects). The concentration of paramagnetic defects increases as the concentration of nanotube is increased (see Fig. 5, right axis). Hence, these defects are localized either within CNTs $\left(\mathrm{Fe}^{3+}\right.$ ions or local defects) or at the CNTs-polymer interface. The extremely low concentration of these defects and the strong interactions between localized and delocalized electrons prevented us from decoupling these contributions.

\section{CONCLUSIONS}

ESR investigations on SIS-CNT composites were reported. An intense symmetrical spectrum with a linewidth of the order of $10^{2} \mathrm{G}$ was noticed at about $g=2.06$ and assigned to strongly interacting localized and itinerant electrons (delocalized over the conducting domains of CNTs) in the bottleneck regime. The temperature dependence of the $g$-factor, of the resonance linewidth, and of the double integral of the resonance spectrum provided compelling evidence for this interpretation. The temperature dependence of the double integral of ESR spectra of CNT suggested that both localized and delocalized electrons reside on CNTs or on the CNT-macromolecular chain interface. The temperature dependence of the resonance linewidth revealed that the thermally induced narrowing of the resonance spectra is largely due to CNTs collisions. These collisions may be responsible for the formation of localized paramagnetic defects.

This work has been supported by US Army STTR grant \#A2-1299 awarded to Indiana University and PartTec Ltd. C.S. Du and N. Pan would like to acknowledge the support by UC Discover grant \# 10,175 .

\section{REFERENCES AND NOTES}

1. Coleman, J. N.; O’Brien, D. F.; Dalton, A. B.; McCarthy, B.; Lahr, B.; Barklie, R. C.; W. J. Blau, W. J. J Chem Phys 2000, 113, 9688-9793.

2. Chipara, M.; Iacomi, F.; Zaleski, J. M.; Bai, J. B. J. Optoelectronics Adv Mat submitted for publication.

3. Chauvet, O.; Baumgartner, G.; Carrard, M.; Bacsa, W.; Ugarte, D.; deHeer, W.A.; Forro, L. Phys Rev B 1996, 53, 13996-13999.

4. Chen, Y.; Chen, J.; Hu, H.; Hamon, M. A.; Itkis, M. E.; Haddon, R. C. Chem Phys Lett 1999, 299, 532-535.

5. Thess, A.; Lee, R.; Nikolaev, P.; Dai, H.; Petit, P.; Robert, J.; Xu, C.; Y.; Lee, Y. H.; Kim, S. G.; Rinzler, A. G.; Colbert, D. T.; Scuseria, G. E.; Tomanek, D.; Fisher, J. E.; Smalley, R. E. Science 1996, 273, 483-487. 
6. Garaj, S.; Thien-Nga, L.; Gaal, R.; Forro, L.; Takahashi, K.; Kokai, F.; Yudasaka, M.; Iijima, S. Phys Rev B 2000, 62, 17115-17119.

7. Yokomichi, H. Vacuum 2004, 74, 677-681.

8. Bandow, S. J Appl Phys 1996, 80, 1020-1027.

9. Duclaux, L.; Salvetat, J. P.; Lauginie, P.; Cacciaguera, T.; Faugere, A. M.; Goze-Bac, C.; Bernier, P. J Phys Chem Solids 2003, 64, 571-581.

10. Goze-Bac, C.; Latil, S.; Lauginie, P.; Jourdain, V.; Conard, J.; Duclaux, L.; Rubio A.; Bernier, P. Carbon 2002, 40, 1825-1842.

11. Ishii, S.; Miyamoto, K.; Oguri, N.; Horiuchi, K.; Sasaki, T.; Aoki, N.; Ochiai, Y. Physica E 2003, 19, 149-152.

12. Kosaka, M.; Ebbesen, T. W.; Hiura, H.; Tanigaki, K. Chem Phy Lett 1995, 233, 47-51.

13. Ochiai, Y.; Enomoto, R.; Ishii, S.; Miyamoto, K.; Matsunaga, Y.; Aoki, N. Physica B 2002, 323, 256-258.

14. Zhang, H.; Liu, S.; Wei, A.; He, Y.; Tang, X.; Xue, X.; Liang, L.; Wu, C. J Phys Chem Solids 2000, 61, 1123-1125.

15. Giraudet, J.; Dubois, M.; Claves, D.; Pinheiro, J. P.; Schouler, M. C.; Gadelle, P.; Hamwi, A. Chem Phys Lett 2003, 381, 306-314.

16. Seelan, S.; Hwang, D.W.; Hwang, L.-P.; Sinha, A. K. Vacuum 2004, 75, 105-109.
17. Petit, P.; Jouguelet, E.; Fischer, J. E.; Rinzler, A. G.; Smalley, R. E. Phys Rev B 1997, 56, 9275-9278.

18. Beuneu, F.; l'Huillier, C. J.-P.; Salvetat, J.-P.; Bonard, J.-M.; Forro, L. Phys Rev B 1999, 59, 5945-5949.

19. Yu, M.-F.; Files, B. S.; Arepalli, S.; Ruoff, R. S. Phys Rev Lett 2000, 84, 5552-5555.

20. Goze, G.; Vaccarini, L.; Henrard, L.; Bernier, P.; Hernandez, E.; Rubio, A. Synth Met 1999, 103, 2500-2501.

21. Allaoui, A.; Bai, S.; Cheng, H. M.; Bai, J. B. Compos Sci Technol 2002, 62 1993-1998.

22. Chauvet, O.; Forro, L.; Bacsa, W.; Ugarte, D.; Doudin, B.; DeHeer, W. A. Phys Rev B 1995, 52, R6963-R6965.

23. Lu, K. L.; Lago, R. M.; Chen, Y. K.; Green, M. L. H.; Harris, P. J. F.; Tsang, S. C. Carbon 1996, 34, 814-816.

24. Poole, C. P.; Farach, H. A. Theory of Magnetic Resonance. 2nd ed.; Wiley: New York, 1987.

25. Chipara, M.; Hui, D.; Sankar, J.; Leslie-Pelecky, D.; Bender, A.; Yue, L.; Skomski, R.; Sellmyer, D. J. Composites B 2004, 35, 235-243.

26. Schneider, H. A. Polymer 2005, 46, 2230-2237.

27. Amama, P. B.; Lim, S.; Ciuparu, D.; Yang, Y.; Pfefferle, L.; Haller, G. L. J Phys Chem B 2005, 109, 2645-2656. 